\title{
Synthesis of a Passive Complex Filter Using Transformers
}

\author{
Kazuhiro Shouno, Member, IEEE, and Yukio Ishibashi
}

\begin{abstract}
In this paper, the synthesis of a passive complex filter has been described. First, a normalized real coefficient filter is transformed into a complex coefficient filter, which includes imaginary-valued resistors by using a proposed frequency transformation. Second, a complex filter using passive elements is presented. The proposed circuit consists of terminating resistors, capacitors, and transformers with finite self-inductances and coupling coefficients equal to or less than unity. A third-order complex Chebyshev bandpass filter is designed using practical passive elements, and its frequency response is measured. It is shown that the experimental circuit exhibits bandpass characteristics $(90-110 \mathrm{kHz})$ and an image rejection of $53 \mathrm{~dB}$.
\end{abstract}

Index Terms-Bandpass filter (BPF), complex, filter, passive, transformer.

\section{INTRODUCTION}

A $\mathrm{N}$ ANALOG filter is one of the most important building blocks in communication systems. The most fundamental filter is an $L C$ filter. Since it exhibits a low noise property and provides a desirable frequency response over a wide frequency range, it is widely used in the front-end circuits of radio systems. However, the inductor in an $L C$ filter is unsuitable for integration. In particular, the element value of the inductor becomes high when the operation frequency of the $L C$ filter is relatively low. To solve this problem, many $R C$-active filters have been proposed [1]-[3]. An $R C$-active filter has a cascade configuration of the second-order sections such as the Sallen-Key circuit [1], Biquad circuit [2], and so on. This structure is employed because the number of active elements in it is relatively small. The problem with this structure is that a high-order filter has high sensitivity. Another method is to simulate a doubly terminated $L C$ filter by using an $R C$-active circuit; this type of filter can inherit the low-sensitivity property of the prototype $L C$ filter [3]. The synthesizing theory of an $L C$ filter has been accomplished; however, it remains a fundamental theory for modern active filter design.

The above-mentioned filter is a real coefficient filter (real filter) whose frequency response is symmetrical with respect to dc $(\omega=0)$. Recently, many techniques with regard to complex coefficient filters (complex filters) have been proposed in order to realize high-performance signal processing. This complex filter has two input terminals: real and imaginary input terminals. As a result, the complex filter has an unsym-

Manuscript received October 12, 2006; revised April 13, 2007. First published February 7, 2008; last published August 13, 2008 (projected). This paper was recommended by Associate Editor A. Kummert.

The authors are with the Graduate School of Systems and Information Engineering, University of Tsukuba, Ibaraki 305-8573, Japan (e-mail: shouno@ cs. tsukuba.ac.jp).

Digital Object Identifier 10.1109/TCSI.2008.918005 metrical frequency response with respect to dc. This property is important for applications in the field of communication systems, e.g., orthogonal communication systems, such as orthogonal modulation/demodulation, image rejection filters, and so on. Many methods have been proposed for designing complex filters [4]-[13]. A polyphase filter [4], [5] is suitable for integrated circuits since it has only capacitors and resistors. However, the resistors lead to power dissipation. As a result, the $R C$ polyphase network has a comparatively high transmission loss.

A frequency-shifting method [6], [8]-[13] simply shifts the frequency response of the prototype filter along the direction of the frequency axis. As a result, the frequency response is symmetrical with respect to the shifting frequency [6]. Extended frequency transformations [7] convert the frequency axis of the prototype filter to that of a complex filter by using a bilinear function. These methods generate an imaginary valued resistor called an imaginary resistor. Its resistance is a constant, pure, and imaginary value, regardless of the frequency; in other words, it is not a practical component. Therefore, the most important problem is to equivalently realize an imaginary resistor by using practical components. An imaginary resistor can be equivalently realized by using a gyrator [12]. However, it is difficult to realize a gyrator using practical passive elements because of its reciprocity. An imaginary resistor can also be equivalently realized by using ideal transformers [13]. In this paper, the frequency-shifting method is used to design the prototype filter. Unfortunately, the resulting complex filter requires an ideal transformer-a transformer with infinite self-inductance. Therefore, it is also difficult to realize this filter by using practical passive components. As described above, most of the conventional complex filters inevitably require active elements. However, the investigation of passive complex filters is important from the viewpoint of their active realization in the same way as conventional real filters.

This paper proposes complex filters using practical passive components. The proposed frequency transformation method is suitable for passive realization. The resulting complex filter includes terminating resistors, capacitors, and transformers with finite self-inductances. Further, as a design example, a third-order complex Chebyshev bandpass filter (BPF) is designed using practical passive elements, and its frequency response is measured.

\section{Design Methodology}

\section{A. Proposed Frequency Transformation}

Conventionally, a real BPF is designed by using a low-pass filter (LPF)-BPF transformation. In this technique, the frequency axis of a normalized real LPF is converted to that of 


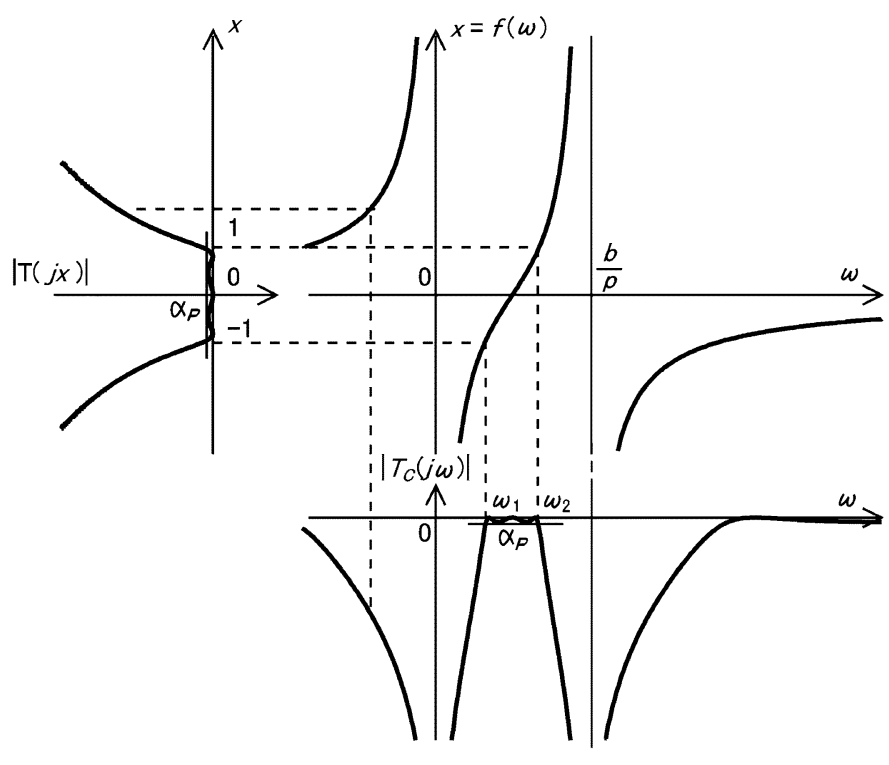

Fig. 1. Proposed transformation.

a real BPF. The proposed complex filter is also designed by using a frequency transformation. Fig. 1 shows the proposed frequency transformation. In this figure, the frequency axis $x$ of the real filter is converted to that of the complex filter. Because the proposed filter is obtained only by frequency transformation, the passband ripple $\alpha_{P}$ of the proposed filter is the same as that of the normalized real LPF. The function $f(\omega)$ is defined as

$$
\begin{aligned}
x & =f(\omega) \\
& =-a\left(\frac{1}{\omega}+\frac{1}{p \omega-b}\right) \\
p & =\frac{1-k^{2}}{k^{2}}
\end{aligned}
$$

where $a>0, b$ is a real constant, and $k$ is the coupling coefficient of the transformer. For the practical transformer, $k$ may be greater than 0.9. In particular, the value of $k$ for the transformer used in the experiment described in Section III is approximately 0.94 . Equation (2) is introduced to simplify the synthesis of the complex filter. Therefore, $k$ and $p$ are the values obtained from the components included in the resulting complex filter. From Fig. 1, the following simultaneous equations can be obtained:

$$
\left.\begin{array}{c}
-1=-a\left(\frac{1}{\omega_{1}}+\frac{1}{p \omega_{1}-b}\right) \\
1=-a\left(\frac{1}{\omega_{2}}+\frac{1}{p \omega_{2}-b}\right)
\end{array}\right\}
$$

For $a>0$, we have

$$
\left.\begin{array}{l}
a=\frac{\left(2 \omega_{G}^{2}-p \omega_{D}^{2}\right)+\sqrt{4 \omega_{G}^{4}+p^{2} \omega_{D}^{2}\left(\omega_{D}^{2}+4 \omega_{G}^{2}\right)}}{2(p+1) \omega_{D}} \\
b=\left(\frac{\omega_{H}}{2}+p \omega_{0}\right)+\frac{1}{2} \sqrt{\omega_{H}^{2}+p^{2} \omega_{D}^{2}}
\end{array}\right\}
$$

where $\omega_{0}=\left(\omega_{1}+\omega_{2}\right) / 2, \omega_{G}^{2}=\omega_{1} \omega_{2}, 2 / \omega_{H}=1 / \omega_{1}+$ $1 / \omega_{2}$, and $\omega_{D}=\omega_{2}-\omega_{1}$. Fig. 2(a) shows the normalized real LPF. The transfer function $T_{C}(j \omega)$ of the resulting complex

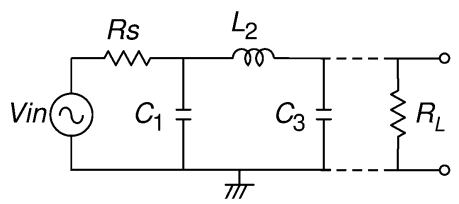

(a)

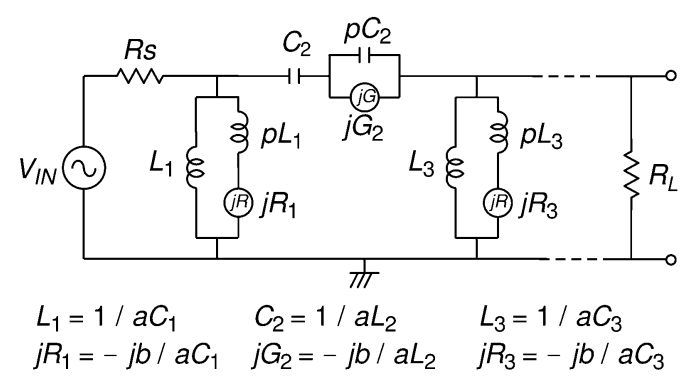

\begin{tabular}{|c|c|}
\hline Reference element & Transformed element \\
\hline$c \underset{0}{1}$ & $1 / a C \xi\left\{\begin{array}{l}\{p / a C \\
(j R)-j b / a C\end{array}\right.$ \\
\hline - & م-jb/aL \\
\hline
\end{tabular}

(b)

Fig. 2. (a) Normalized real LPF. (b) Complex filter.

TABLE I

ELEMENT TRANSFORMATION

filter becomes $T_{C}(j \omega)=T(j f(\omega))$. The inductor $L$ included in the normalized real LPF gets transformed to

$$
Z_{L}(j \omega)=j x L=\frac{1}{j \omega \frac{1}{a L}}+\frac{1}{j \omega \frac{p}{a L}-j \frac{b}{a L}} .
$$

The admittance $Y_{C}(j \omega)$ of the capacitor $C$ becomes

$$
Y_{C}(j \omega)=j x C=\frac{1}{j \omega \frac{1}{a C}}+\frac{1}{j \omega \frac{p}{a C}-j \frac{b}{a C}} .
$$

In these equations, $j$ is an imaginary unit. Table I summarizes the element transformations given by (5) and (6). From Table I, it is found that the resulting complex filter includes the element with an impedance of either $j R$ or $j G$. It is referred to as an imaginary resistor. The imaginary resistor has a purely imaginary resistance, regardless of the frequency $\omega$. Therefore, this element is not a practical element. The prototype filter shown in Fig. 2(a) is transformed to the complex filter shown in Fig. 2(b) through the proposed frequency transformation. 


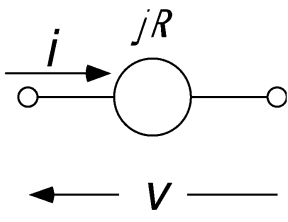

(a)

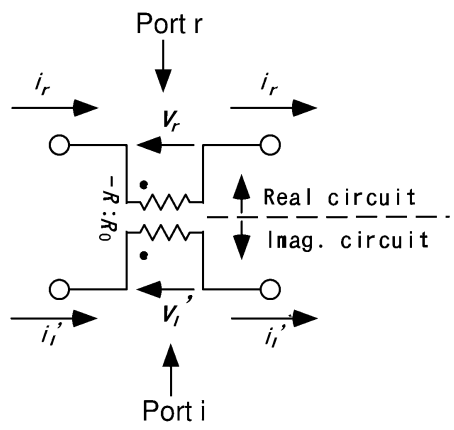

(b)

Fig. 3. (a) Imaginary resistor. (b) Imaginary resistor realized by using an ideal transformer.

\section{B. Imaginary Resistor}

The circuit equation of the imaginary resistor $j R$ shown in Fig. 3(a) has the following form:

$$
v=j R i \text {. }
$$

The voltage $v$ and current $i$ can be decomposed into their real and imaginary components

$$
\left.\begin{array}{l}
v=v_{r}+j v_{i} \\
i=i_{r}+j i_{i}
\end{array}\right\}
$$

The subscripts $r$ and $i$ denote the real and imaginary signal paths, respectively. This means that the complex filter has real and imaginary circuits. Substituting (8) into (7) yields

$$
\left(\begin{array}{c}
v_{r} \\
v_{i}
\end{array}\right)=\left(\begin{array}{cc}
0 & -R \\
R & 0
\end{array}\right)\left(\begin{array}{c}
i_{r} \\
i_{i}
\end{array}\right)
$$

When this equation is regarded as a $Z$-matrix, which indicates the relationship between the ports $\mathrm{r}$ and $\mathrm{i}$, its configuration reveals that the imaginary resistor can be equivalently realized by using a gyrator with a gyration resistance of $R$ [12]. Now, we introduce $v_{i}^{\prime}$ and $i_{i}^{\prime}$ defined as follows:

$$
\left.\begin{array}{l}
v_{i}^{\prime}=R_{0} i_{i} \\
i_{i}^{\prime}=\left(1 / R_{0}\right) v_{i}
\end{array}\right\}
$$

where $R_{0}$ is a positive constant. It should be noted that (10) implies that the current and voltage are interchanged in the imaginary circuit. Therefore, the imaginary circuit must represent a dual of the real circuit. Substituting (10) into (9) yields

$$
\left(\begin{array}{c}
v_{r} \\
i_{i}^{\prime}
\end{array}\right)=\left(\begin{array}{cc}
0 & -R / R_{0} \\
R / R_{0} & 0
\end{array}\right)\left(\begin{array}{l}
i_{r} \\
v_{i}^{\prime}
\end{array}\right) .
$$

This equation is in the $h$-matrix form. Rewriting it in an $F$-matrix form yields

$$
\left(\begin{array}{c}
v_{r} \\
i_{r}
\end{array}\right)=\left(\begin{array}{cc}
-R / R_{0} & 0 \\
0 & -R_{0} / R
\end{array}\right)\left(\begin{array}{c}
v_{i}^{\prime} \\
-i_{i}^{\prime}
\end{array}\right) .
$$

It should be noted that the sign of $i_{i}^{\prime}$ is inverted since the current direction of the $F$-matrix is opposite to that of the $h$-matrix. Equation (12) represents an ideal transformer embedded between the real and imaginary circuits. Therefore, the imaginary resistor shown Fig. 3(a) can be equivalently realized by using the ideal transformer whose turn ratio is $-R: R_{0}$ as shown in Fig. 3(b) [13].

The dual circuit [14] shown in Fig. 4(b) can be obtained as follows. When the relationship between two elements $Z_{1}$ and $Z_{2}$ is given by $Z_{1} Z_{2}=R_{0}^{2}$, they act as dual elements, as listed in Table II. The dual of the original circuit shown in Fig. 4(a) can be obtained using the following procedure.

Step 1) Place nodes 1-6 inside each loop and node 7 outside the real circuit.

Step 2) Draw dotted lines connecting two nodes through the elements in the original circuit.

Step 3) Assign the elements traversed by the dotted lines to their dual elements, as listed in Table II.

Fig. 4(b) shows the resulting dual circuit. The ideal transformers $\mathrm{T}_{1}-\mathrm{T}_{3}$ couple the real and imaginary circuits. The polarities of transformer $\mathrm{T}_{1}$ can be determined through the following procedure.

Step 1) Assume that the current flows in the clockwise direction around node 2, as shown in Fig. 4(a). ${ }^{1}$ We place a node on the side of the coil from which current flows inward.

Step 2) The polarity of the secondary winding is assumed such that current flows into node 2 of the dual circuit. This means that we place a node on the side of the coil from which the current flows outward.

The above procedure can be applied to the other ideal transformers $T_{2}$ and $T_{3}$ for nodes 4 and 5 . A combination of the circuit shown in Fig. 4(a) with that shown in 4(b) yields the complex filter shown in Fig. 5. In this figure, $R_{0}$ is set to unity for simplicity. For $R_{S}=1$, the input resistances $R_{S}$ of the real circuit is the same as $1 / R_{S}$ of the imaginary circuit. ${ }^{2}$ Under this condition, the amplitude of $V_{\mathrm{IMAG}} / R_{S}$ is the same as that of $V_{\text {REAL }}$.

\section{Loosely Coupled Transformer}

Fig. 6(a) shows the ideal transformer with the surrounding elements used in the complex filter shown in Fig. 5. The impedances $Z_{A 1}$ and $Z_{A 2}$ and the transfer gain $V_{A 2} / V_{A 1}$ can be represented as

$$
\left.\begin{array}{l}
Z_{A 1}=s L \\
Z_{A 2}=s n^{2} L(p+1) \\
V_{A 2} /\left.V_{A 1}\right|_{I_{A 2}=0}=n
\end{array}\right\}
$$

Fig. 6(b) shows the equivalent circuit of the loosely coupled transformer; $M$ represents the mutual inductance. From this figure, we have

\footnotetext{
${ }^{1}$ When we select node 3 instead of node 2 , the resulting polarity remains unchanged.

${ }^{2}$ Usually, the terminating resistor $R_{S}$ of the normalized filter is unity.
} 


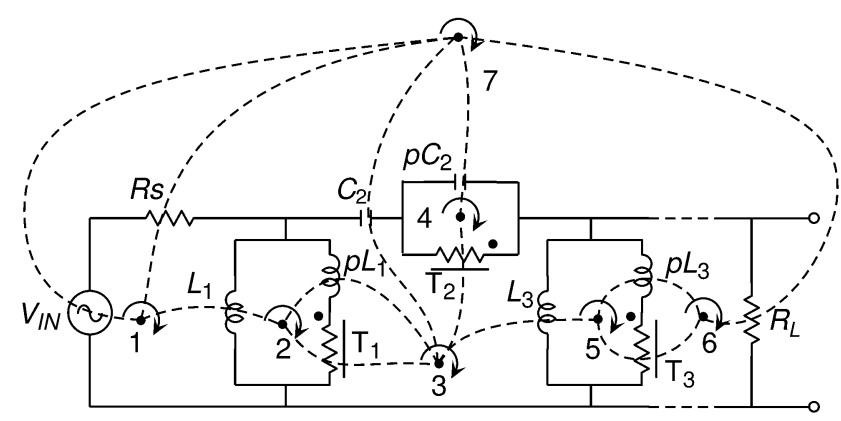

(a)

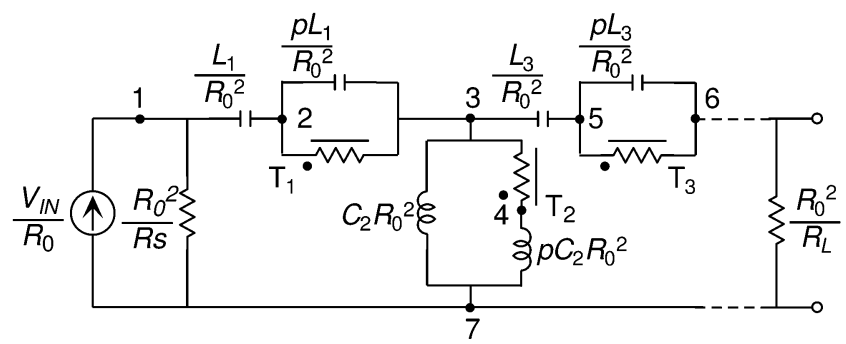

(b)

Fig. 4. (a) Original circuit. (b) Dual circuit.

TABLE II

DUAL ELEMENTS
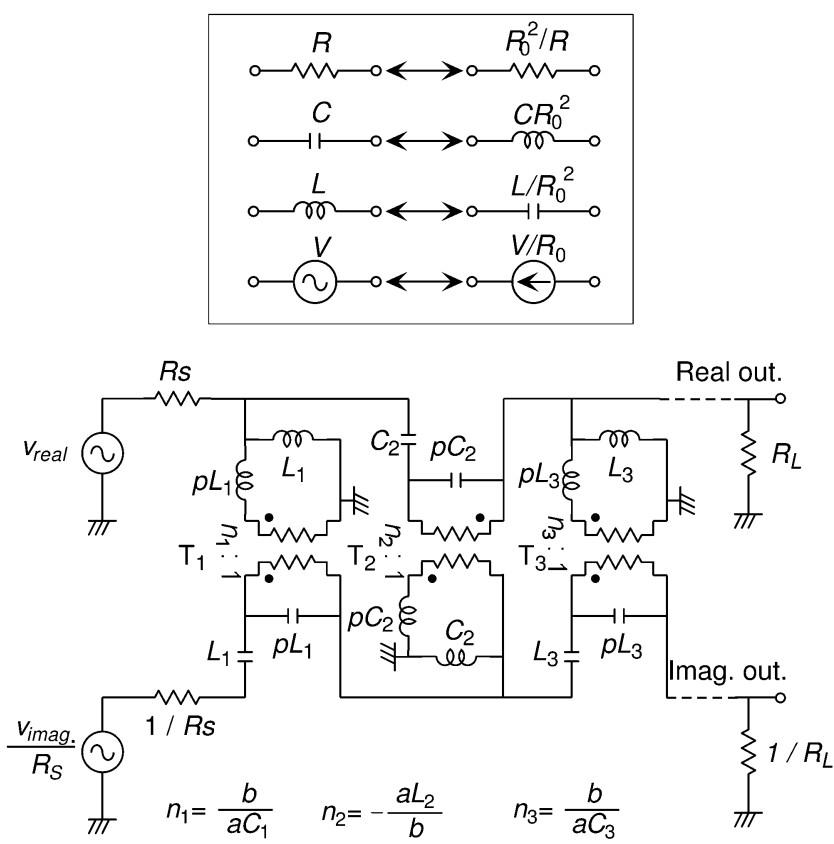

Fig. 5. Complex filter using ideal transformers.

$$
\left.\begin{array}{l}
Z_{B 1}=s L_{1} \\
Z_{B 2}=s n^{2} L_{2} \\
V_{B 2} / V_{B 1}=M / L_{1} .
\end{array}\right\}
$$

When $Z_{A 1}=Z_{B 1}, Z_{A 2}=Z_{B 2}$, and $V_{A 2} / V_{A 1}=V_{B 2} / V_{B 1}$ are satisfied, they become equivalent to each other. Therefore, we have

$$
\left.\begin{array}{l}
L_{1}=L \\
L_{2}=n^{2} L(1+p) \\
M=n L .
\end{array}\right\}
$$

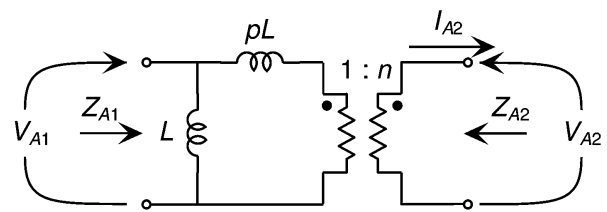

(a)

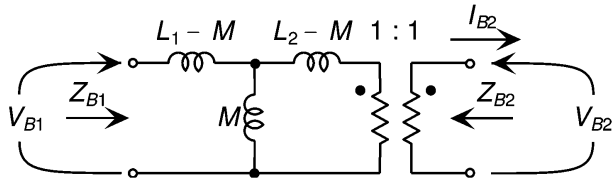

(b)

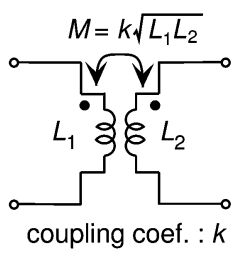

(c)

Fig. 6. (a) Ideal transformer and its surrounding elements. (b) Equivalent circuit of loose coupling transformer. (c) Loosely coupled transformer.

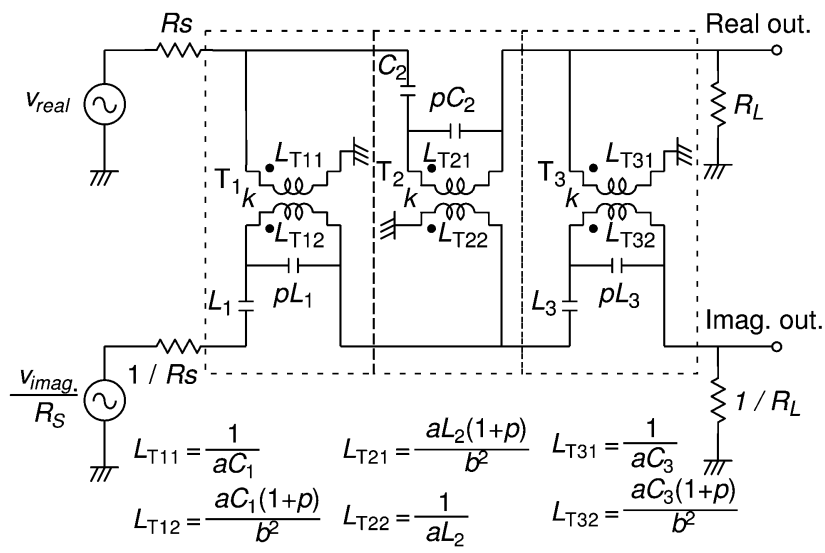

Fig. 7. Proposed circuit $(n=3)$.

On the basis of the above assumptions, it is found that the circuit shown in Fig. 6(a) is equivalent to the circuit shown in Fig. 6(c). Therefore, as shown in Fig. 7, the proposed complex filter can be realized by using the loosely coupled transformer.

Equation set (15) implies that all of the transformers included in the proposed circuit have the identical mutual inductance $M=R_{0} / b$. When a high-order filter is designed, a complex filter can be realized by cascading the building blocks surrounded by the broken lines in Fig. 7 .

\section{Design EXAmple}

First, a complex filter that satisfies the following specifications is designed.
A third-order complex
Passband ripple
$\mathrm{BPF}(n=3)$.
Passband edges
$1 \mathrm{~dB}$.
Coupling coefficient
$\omega_{1}=9 \mathrm{rad} / \mathrm{s}$ and
$\omega_{2}=11 \mathrm{rad} / \mathrm{s}$.
$k=0.94$. 
TABLE III

ELEMENT VALUES

\begin{tabular}{|c|c|c|c|c|}
\hline Element & Theoretical & Measured & Turns & Q \\
\hline \hline$R_{S R}$ & $200 \Omega$ & $200.7 \Omega$ & - & - \\
$R_{S I}$ & $200 \Omega$ & $200.8 \Omega$ & - & - \\
$L_{T 11}$ & $18.003 \mu \mathrm{H}$ & $18.08 \mu \mathrm{H}$ & 6 & 58.3 \\
$L_{T 12}$ & $5.0605 \mathrm{mH}$ & $5.080 \mathrm{mH}$ & 104 & 350 \\
$L_{T 21}$ & $2.4860 \mathrm{mH}$ & $2.474 \mathrm{mH}$ & 68 & 390 \\
$L_{T 22}$ & $36.646 \mu \mathrm{H}$ & $36.73 \mu \mathrm{H}$ & 8 & 95.7 \\
$L_{T 31}$ & $18.003 \mu \mathrm{H}$ & $18.01 \mu \mathrm{H}$ & 6 & 50.7 \\
$L_{T 32}$ & $5.0605 \mathrm{mH}$ & $5.076 \mathrm{mH}$ & 105 & 340 \\
$L_{1}$ & $450.06 \mathrm{pF}$ & $450.1 \mathrm{pF}$ & - & $>1000$ \\
$p L_{1}$ & $59.289 \mathrm{pF}$ & $59.39 \mathrm{pF}$ & - & $>1000$ \\
$C_{2}$ & $916.16 \mathrm{pF}$ & $916.1 \mathrm{pF}$ & - & $>1000$ \\
$p C_{2}$ & $120.69 \mathrm{pF}$ & $120.5 \mathrm{pF}$ & - & $>1000$ \\
$L_{3}$ & $450.06 \mathrm{pF}$ & $450.0 \mathrm{pF}$ & - & $>1000$ \\
$p L_{3}$ & $59.289 \mathrm{pF}$ & $59.32 \mathrm{pF}$ & - & $>1000$ \\
$R_{L R}$ & $200 \Omega$ & $199.9 \Omega$ & - & - \\
$R_{L I}$ & $200 \Omega$ & $200.0 \Omega$ & - & - \\
\multicolumn{5}{|r|}{}
\end{tabular}

The circuit configuration of the normalized LPF is the same as that in Fig. 2(a). In this figure, $R_{S}=R_{L}=1, C_{1}=C_{3}=$ 2.0236, and $L_{2}=0.9941$ [15]. Substituting $k=0.94$ into (2) yields $p=0.13173$. Substituting $\omega_{1}=9 \mathrm{rad} / \mathrm{s}$ and $\omega_{2}=11$ $\mathrm{rad} / \mathrm{s}$ in (4) yields

$$
\left.\begin{array}{l}
a=87.376 \\
b=11.219 .
\end{array}\right\}
$$

The element values shown in Figs. 2(b), 5, and 7 become

$$
\begin{aligned}
& R_{S}=R_{L}=1 \\
& L_{1}=L_{3}=L_{\mathrm{T} 11}=L_{\mathrm{T} 31}=0.0056557 \\
& p L_{1}=p L_{3}=0.00074505 \\
& j R_{1}=j R_{3}=-0.063452 j \\
& C_{2}=L_{\mathrm{T} 22}=0.011513 \\
& p C_{2}=0.0015166 \\
& j G_{2}=-0.12916 j \\
& n_{1}=n_{3}=0.063452 \\
& n_{2}=-7.7422 \\
& L_{\mathrm{T} 12}=L_{\mathrm{T} 32}=1.5898 \\
& L_{\mathrm{T} 21}=0.78100 .
\end{aligned}
$$

Second, the element values of the experimental circuit are listed in Table III. The element values are scaled such that $\omega_{1}, \omega_{2}$, and the impedance level become $90 \mathrm{kHz}, 110 \mathrm{kHz}$, and $200 \Omega$, respectively. The deviations between the desired and measured element values are within $0.5 \%$. Fig. 8 shows the transformer constructed for experimental purposes. As shown in this figure, the transformer is realized by using a ferrite pot core. In order to reduce the coupling capacitance between the primary and secondary windings, the bobbin is equipped with a separator. The bobbin is a Delrin rod shaped using a turning lathe. As a result, the coupling capacitance is reduced. The measured value of $k$ is approximately 0.94 . Fig. 9 shows the experimental results obtained by the measurement technique described in [16], [17]. The experimental results show that the proposed filter exhibits a complex bandpass response and an image rejection of $53 \mathrm{~dB}$. It is found that the deviation between the measured and ideal frequency responses is caused due to the parasitic elements. In this figure, the parasitic elements include the dissipation and coupling capacitances of the transformers.

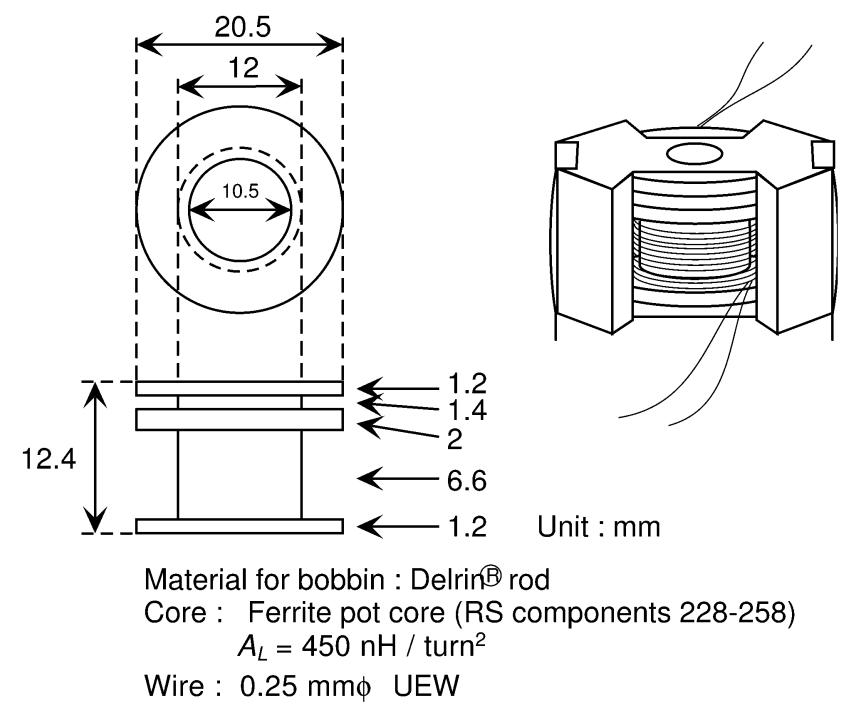

Fig. 8. Transformer constructed for experiment.

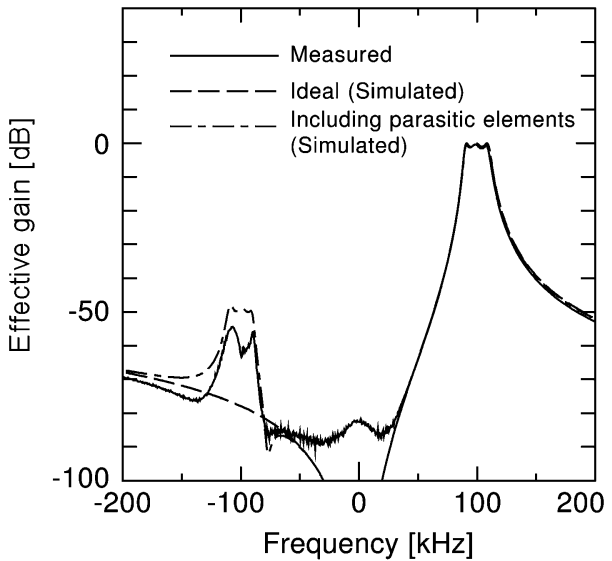

(a)

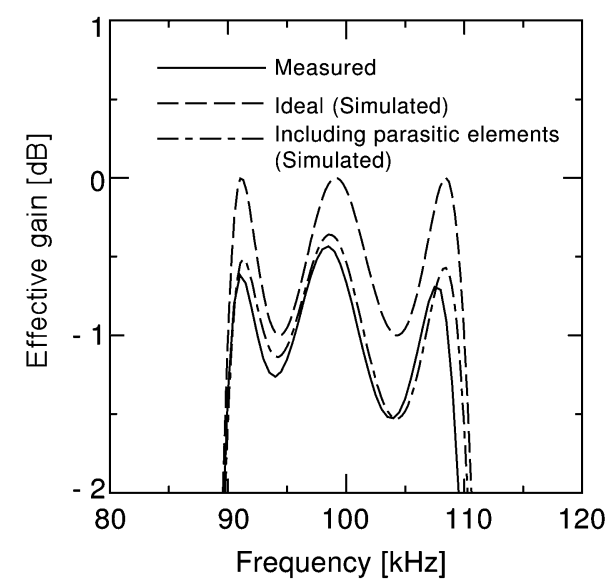

(b)

Fig. 9. Experimental results. (a) Overall. (b) Enlargement near passband.

Fig. 10 shows the frequency characteristics for $k=$ $0.9,0.7,0.5$, and $0.5(n=5)$. The specifications are the same as those given above, except for the value of $k$. This figure shows that the value of $k$ influences the frequency characteristics. By using the proposed method, the complex filter that includes a loosely coupled transformer with $k$ satisfying 


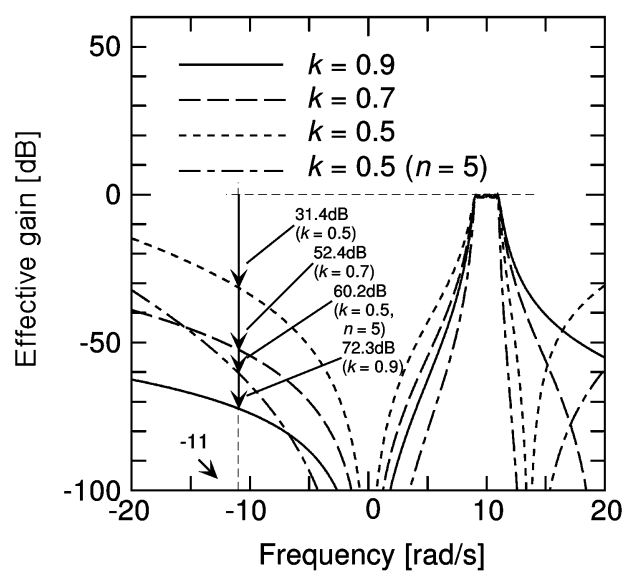

Fig. 10. Simulated frequency response for $k=0.9,0.7,0.5$ and $0.5(n=5)$.

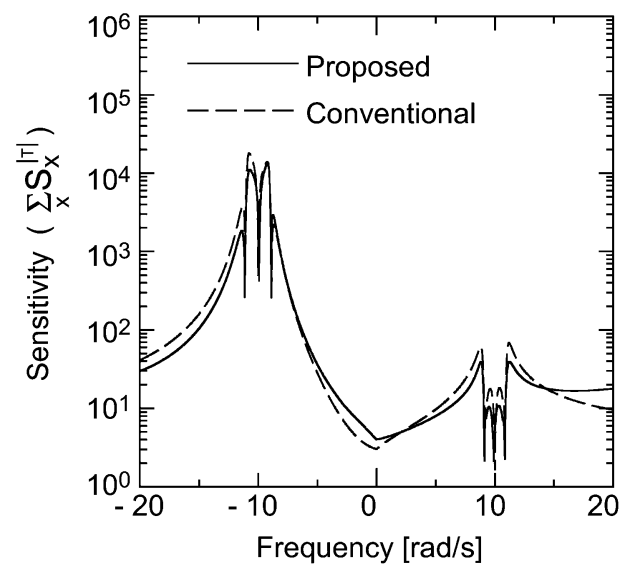

Fig. 11. Sensitivity.

$0<k \leq 1$ can be designed. Generally, from the viewpoint of application purposes, it is desirable that the image rejection be as large as possible. Fig. 10 shows that the lower the value of $k$, the smaller the image rejection. Therefore, it is desirable to use a transformer with the highest possible value of $k$. If it is difficult to use such a transformer, then a higher order normalized real low-pass prototype filter can be used.

Fig. 11 shows the calculated results of the worst case sensitivities of the gain to the variation of the elements included in the proposed and the conventional circuits [13]. It should be noted that the conventional circuit cannot be realized by using only the practical passive components because ideal transformers are required. From Fig. 11, it is found that both the circuits have similar sensitivity characteristics. In particular, for the negative frequencies near $-100 \mathrm{kHz}$, they possess very high sensitivity. This implies that the proposed method also requires very small element tolerances and the absence of noticeable parasitic elements when high image rejection is required.

\section{CONCLUSION}

In this paper, a passive complex filter has been proposed. The proposed circuit is obtained by using a frequency transformation technique different from the conventional frequency-shifting method. The proposed frequency transformation is suitable for passive realization. The resulting complex filter includes passive components, namely, transformers, capacitors, and terminating resistors.

As an example, a third-order complex filter has been designed. Since the value of $k$ influences the image rejection, it is desirable that we use a transformer with the highest possible value of $k$ from the viewpoint of its manufacturing cost. The transformer included in the experimental circuit is realized using a ferrite pot core. The transformer functions as an imaginary resistor including the surrounding inductors; the combination of this transformer and the surrounding capacitors can be used to realize the proposed complex filter. The proposed method has been validated through experiments. The experimental results are in good agreement with the simulated frequency characteristics. Further investigation is required to eliminate the transformers from the passive complex filter.

\section{REFERENCES}

[1] R. P. Sallen and E. L. Key, "A practical method of designing $R C$ active filters," IRE Trans. Circuit Theory, vol. CT-2, pp. 75-85, Mar. 1955.

[2] L. C. Thomas, "The biquad, Part 1-Some practical design considerations," IEEE Trans. Circuit Theory, vol. CT-18, pp. 350-357, May 1971.

[3] H. J. Orchard, "Inductorless filters," Electron. Lett., vol. 2, no. 6, pp. 224-225, Jun. 1966.

[4] M. J. Gingell, "The synthesis and application of polyphase filters with sequence asymmetric properties," Ph.D. dissertation, Faculty of Engineering, Univ. of London, London, U.K., 1975.

[5] J. Crols and M. Steyaert, "A single-chip $900 \mathrm{MHz}$ CMOS receiver front-end with a high performance low-IF topology," IEEE J. SolidState Circuits, vol. 30, no. 12, pp. 1483-1492, Dec. 1995.

[6] A. S. Sedra, W. M. Snelgrove, and R. Allen, "Complex analog bandpass filters desgined by linearly shifting real lowpass prototypes," in Proc. IEEE Int. Symp. Circuits Syst., 1985, pp. 1223-1226.

[7] C. Muto, "A new extended frequency transformation for complex analog filter design," IEICE Trans. Fundamentals, vol. E83-A, no. 6, pp. 934-940, Jun. 2000.

[8] C. Muto and H. Hoshikawa, "An integrable image rejection system using a complex analog filter with variable bandwidth and center frequency characteristics," IEICE Trans. Fundamentals, vol. E85-A, no. 2, pp. 309-315, Feb. 2002.

[9] M. Steyart and R. Roovers, "A 1-GHz single-Chip quadrature modulator,” IEEE J. Solid-State Circuits, vol. 27, no. 8, pp. 1194-1197, Aug. 1992.

[10] C. Muto and H. Kambayashi, "A leapfrog synthesis of complex analog filters," IEICE Trans. Fundamentals, vol. E76-A, no. 2, pp. 210-215, Feb. 1993.

[11] B. Guthrie, J. Hughes, T. Sayers, and A. Spencer, "A CMOS gyrator low-IF filter for a dual-mode Bluetooth/ZigBee transceiver," IEEE J. Solid-State Circuits, vol. 40, no. 9, pp. 1872-1879, Sep. 2005.

[12] J. O. Voorman, "Continuous-time analog integrated filters," in Integrated Continuous-Time Filters-Principles, Design, and Applications, Y. P. Tsivids and J. O. Voorman, Eds. New York: IEEE, 1993, pp. 15-46.

[13] K. Shouno and Y. Ishibashi, "Synthesis of a complex coefficient filter by a passive elements including ideal transformers and its simulation using operational amplifiers," IEICE Trans. Fundamentals, vol. E83-A, no. 6, pp. 949-955, Jun. 2000.

[14] M. E. Van Valkenburg, Network Analysis. Englewood Cliffs, NJ: Prentice-Hall, 1964, pp. 77-80.

[15] M. E. Van Valkenburg, Analog Filter Design. New York: Oxford Univ. Press, 1982, p. 414.

[16] K. Shouno and Y. Ishibashi, "A note on the measurement method of the frequency response of a complex coefficient filter," IEICE Trans. Fundamentals, vol. J81-A, no. 12, pp. 1486-1494, Dec. 2000.

[17] K. Shouno and Y. Ishibashi, "A note on the measurement method of the frequency response of a complex coefficient filter," in Electronics and Communications in Japan. New York: Wiley, Feb. 2002, vol. 85, 6, pt. 3, pp. 32-41, (English version of [16]). 


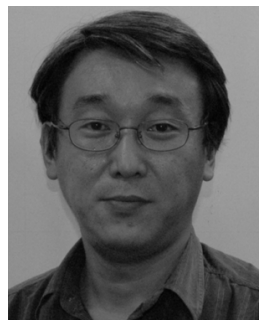

Kazuhiro Shouno (M'04) was born in Tokushima, Japan, in 1971. He received the B.E., M.E., and D.E. degrees in engineering from the University of Tsukuba, Ibaraki, Japan, in 1994, 1996, and 2001, respectively.

From 1996 to 1998 , he was with Matsushita Electric Industrial Company, Ltd. From 1998 to 2001, he was engaged in doctoral research. From 2001 to 2003, he was a Research Assistant with Bio-System Engineering, Faculty of Engineering, Yamagata University. Since 2003, he has been with the Institute of the Information Sciences and Electronics, University of Tsukuba, where he is an Assistant Professor. His research interests include passive/active network theory and analog integrated circuits.

Dr. Shouno is a member of the Institute of Electronics, Information and Communication Engineers (IEICE) of Japan and the Institute of Electrical Engineers (IEE) of Japan.

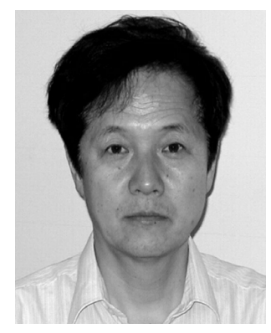

Yukio Ishibashi was born in Tokyo, Japan, in 1945. He received the B.E., M.E., and Dr.E. degrees in electrical engineering from the Tokyo Institute of Technology, Tokyo, Japan, in 1968, 1970, and 1973, respectively.

He was a Research Assistant with the Tokyo Institute of Technology from 1973 to 1980. From 1980 to 1993, he was an Associate Professor with the Institute of Applied Physics, University of Tsukuba. Since 1993, he has been with the Institute of Information Sciences and Electronics, University of Tsukuba, where he is a Professor. His research interests are in the field of active network theory. 